\title{
Numerical of A Call Centre with Two Types of Queue
}

\author{
G. Arul Freeda Vinodhini, R. Ravikumar
}

\begin{abstract}
Call centre is modelled as a queue system with invisible queue and virtual queue. It forms a special type of structured level dependent matrix. Matrix geometric method is used to find the time independent joint distribution of number of invisible customers and visible customers. Through numerical examples we have given the comparison between the existing call centre queue model and the model that we have proposed.
\end{abstract}

Keywords M/M/1 queue - Steady State Probabilities - Matrix Geometric method - Call centre with invisible queue and virtual queue. $90 C 40$ - Secondary 68M20

\section{INTRODUCTION}

All of us experience queues in one form or another in our day to day activities. We need a greater focus to make the large class of queue models developed more directly applicable in the real world $[2,10]$. Our work considers call centre as a queue model consisting of queue with invisible and queue with virtual customers. To achieve best quality of service (QoS) we allow customers of virtual queue to get register themselves and continue with their own work.

Visible queue is a queue where customers physically stand in a queue and hence they know how many customers are ahead of them. But in classic call centre models, calls that arrive are put in a queue. This queue we call it as an invisible queue because customers do not know how much time they have to wait to get their service and how many are ahead of them. To overcome this difficulty now - a - days every unit of time they get an alert telling them about their average waiting time and number of customers ahead of them. This has attracted customers to wait in queue but still their waiting time is not spent in a useful way. Motivated by this fact we introduce another queue called virtual queue where the registered customers are served in FIFO way. These customers can spend their waiting time in their own way until served by a server. This queue is made finite to ensure that all virtual queue customers are server by the end of the day. If it happens that the server is busy the entire day he works overtime to serve the virtual customers. By this way we can make atmost $\mathrm{N}$ customers to spend their waiting time usefully.

This model differs from retrial model because they describe the distinctive nature of customers who repeats his request after a short while in a random fashion. But in our model the customer is not repeating the request instead the server calls him back to finish his service when free. Several

Revised Manuscript Received on 14, October 2019.

G. Arul Freeda Vinodhini, A.P (SG), Saveetha School of Engineering, SIMATS, Chennai, Tamilnadu, India.(E-mail: arulfreedav@gmail.com)

R. Ravikumar, Asst. Prof, Saveetha School of Engineering, SIMATS, Chennai, Tamilnadu, India.(E-mail: dr.ravikumar70@gmail.com)
Mathematics subject Classification (2010) Primary 60K25,

queue papers [3, 4, 6, 7 and 9] have come with two types of customers, but none of them have taken this class of virtual customers. There are few literatures which modeled call centers as retrial queues in [2]. The time independent probabilities of level dependent QBD's are expressed in terms of a sequence of rate matrices as given in $[5,8]$.

The notion of multi-server also differs from other queue models. Whenever a call arrives to the call centre it is diverted to the various servers in rotational basis. We are not bothered whether server is available at that time. This is done to ensure each server receive equal number of virtual customers and equal number of invisible customers. Flow of customers is very large in a call centre and hence we may take the arrival rate of invisible or virtual customer remains the same for each server in the call centre. As each server works heterogeneously their idle times may differ but still they are forced to finish the registered customers before the day ends. Thus a call centre is divided into independent multi stations with a single server each working parallel. The mathematical model that follows is for a station in a call centre. Hence the average number of customers in a call centre is the sum of average number of customers in each station.

Our paper contribution includes introduction to a new class of customers called virtual customers, model it as a level dependent QBD and find the joint stationary distribution with Matrix Geometric Method. Using numerical examples we have shown that this model will attract more customers than the existing models. The rest of our paper is arranged as follows. Mathematical model of a call centre is presented in section 2. The Matrix Geometric Method to find stationary probabilities is explained in section 3. Section 4 gives other performance measures. Numerical examples are discussed in section 5. Conclusion of the work and future directions for development of the proposed model is given in section 6 .

\section{MODEL AND FORMULATION}

\subsection{Model}

We consider a queue model with single server and two types of queues. In queue of type 1[Invisible queue], customers wait in call line to get their service where as in queue of type 2[virtual queue], the customers have registered themselves to get served by the server whenever free in FIFO manner. Let customers arrive to invisible queue and get registered in virtual queue in accordance with Poisson process. Let $\lambda_{1}$ and $\lambda_{2}$ be their arrival rate 
respectively. Let both the type of customers be server in exponential time with mean $1 / \mu$. Only $\mathrm{N}$ customers are allowed to register in virtual queue per day. We assume that whenever invisible queue has no customer the server starts serving virtual customers or become idle if no virtual customers are found. Similarly whenever virtual queue has no customer the server starts serving invisible customers or become idle if no invisible customers are found. Also if the server is continuously busy serving invisible customers at the end of the work time he completes all virtual customers during overtime. To make this meaningful we are allowing only finite number of customers in virtual queue.

\subsection{Level dependent $Q B D$ process}

Let $C(t), N_{1}(t)$ and $N_{2}(t)$ represent server state [0 - idle, 1 - serving virtual customer, 2 - serving invisible customer], the number of customers in virtual queue $[0,1,2,3 \ldots \mathrm{M}]$ and number of customers in invisible queue $[0,1,2,3 \ldots]$ at time t. Letting $X(t)=\left\{C(t), N_{1}(t), N_{2}(t)\right\}$ the trivariate process is a Markov chain $\{X(t), t \geq 0\}$ with state space $S=\{0,1,2\} \times\{0,1,2 \ldots M\} \times Z_{+}$where $Z_{+}=\{0,1,2 \ldots\}$. For positive recurrent process $\{X(t)\}$ the necessary and sufficient condition is given by $\frac{\lambda}{\mu}<1$ where $\lambda=\lambda_{1}+\lambda_{2}$.

The infinitesimal generator $\mathrm{Q}$ of the process $X(t)$ can be expressed as a level dependent process as follows.

$$
Q=\left[\begin{array}{cccccc}
B_{00} & B_{01} & O & O & 0 & \cdots \\
B_{10} & A_{1} & A_{2} & O & 0 & \ldots \\
O & A_{0} & A_{1} & A_{2} & O & \ldots \\
O & O & A_{0} & A_{1} & A_{2} & \ldots \\
\vdots & \vdots & \vdots & \vdots & \vdots & \ddots
\end{array}\right]
$$

where $O$ is the null matrix with suitable dimension, $A_{i}$ are matrices of order $2 \mathrm{M}+1 \times 2 \mathrm{M}+1, \quad B_{00}$ is a matrix of order $\mathrm{M}+1 \times \mathrm{X}+1, B_{01}$ is a matrix of order $(M+1) X(2 M+1)$ and $B_{10}$ is a matrix of order $(2 M+$ 1) $X(M+1)$ given as follows.

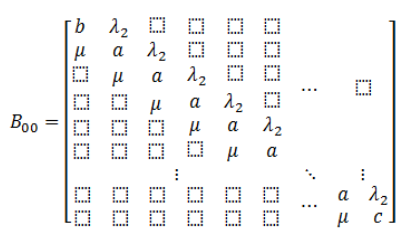

where and $c=-\mu-\lambda_{1}$ $b=-\lambda_{1}-\lambda_{2}$

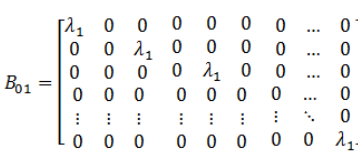

$$
B_{01}=\left[\begin{array}{cccccc}
\mu & 0 & 0 & 0 & \ldots & 0 \\
0 & \mu & 0 & 0 & \ldots & 0 \\
0 & 0 & \mu & 0 & \ldots & 0 \\
0 & 0 & 0 & \mu & \ldots & 0 \\
\vdots & \vdots & \vdots & \vdots & \ddots & 0 \\
0 & 0 & 0 & 0 & \ldots & \mu
\end{array}\right]
$$
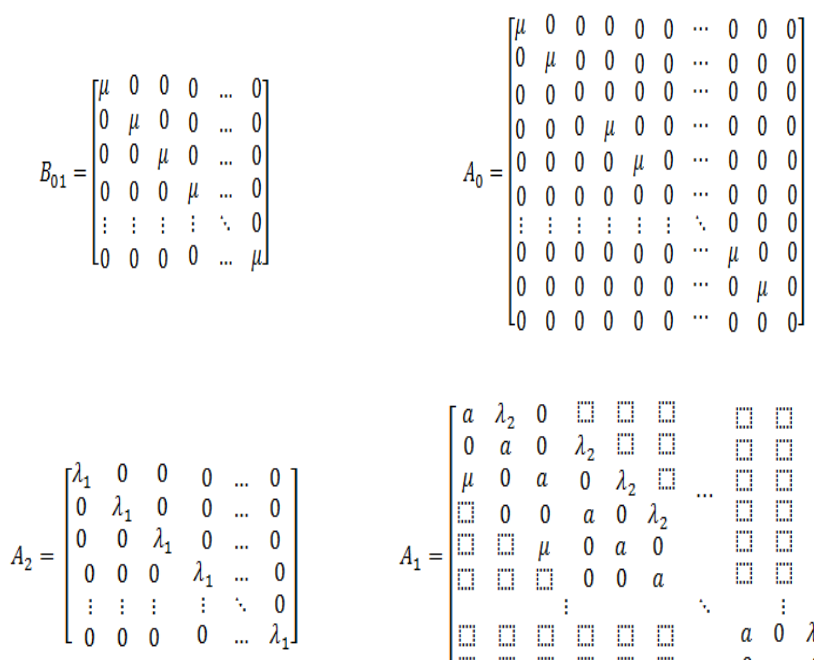

Let

$$
p_{i, j, k}=\lim _{t \rightarrow \infty} P\left[C(t)=i, N_{1}(t)=j, N_{2}(t)=k\right]
$$
denotes joint time independent probability of the server state, number of invisible and visible customers.

Let $\pi=\pi_{0}, \pi_{1}, \pi_{2}, \pi_{3} \ldots$

with $\pi_{0}=\left[\begin{array}{lllll}p_{000} & p_{210} & p_{220} & \ldots & p_{2 M 0}\end{array}\right]$

$\pi_{n}=\left[\begin{array}{llllllll}p_{10 n} & p_{11 n} & p_{21 n} & p_{12 n} & p_{22 n} & \ldots & p_{1 M n} & p_{2 M n}\end{array}\right]$ where $n=1,2, \ldots \infty$

The time independent probability $\pi$ should satisfy the set of equations

$$
\pi Q=0 \quad \pi e=1
$$

Where vector e is a column vector of suitable dimension with all entries as 1 and vector $O$ is a row vector of suitable dimension with all entries as zero.

\section{MATRIX GEOMETRIC METHOD}

The discrete Markov chain $\left\{C(t), N_{1}(t), N_{2}(t), t \geq 0\right\}$ is Ergodic if and only if $\pi_{A} A_{2} e<\pi_{A} A_{0} e$ where $A=A_{1}+$ $A_{2}+A_{3}$ and $\pi_{A} A=0$. Equation (1) can be rewritten as

$$
\begin{gathered}
\pi_{0} B_{00}+\pi_{1} B_{10}=0 \\
\pi_{0} B_{01}+\pi_{1}\left[A_{1}+R A_{0}\right]=0 \\
\pi_{0} e+\pi_{1} \sum_{i=0}^{\infty} R^{i} e=1
\end{gathered}
$$

The solution is given by $\pi_{n}=\pi_{n-1} R^{(n)} \quad n \in \mathbb{N}=$ $\{2,3 \ldots\}$ where $R^{(n)}$ satisfies the equation $\pi_{1} R^{(n-2)} A_{2}+$ $\pi_{1} R^{(n-1)} A_{1}+\pi_{1} R^{(n)} A_{0}=0$.

The Eigen values of $\mathrm{R}$ lie in the interior of a unit circle. Hence (I-R) is non singular. Compute $\sum_{i=0}^{\infty} R^{i}=(I-R)^{-1}$ and $\alpha=\pi_{0} e+\pi_{1}(I-R)^{-1} e$.

Divide the computed sub vector $\pi_{0}$ and $\pi_{1}$ by $\alpha$ to normalize $\pi_{0}$ and $\pi_{1}$.

\section{OTHER PERFORMANCE MEASURES}

(1) The probability that there are zero customers in the invisible queue is given by $\quad \pi_{0 N_{2}}=\sum_{i=1}^{M+1} \pi_{0, i}$

(2) The probability that there are $\mathrm{n}$ customers in the invisible queue is given by $\quad \pi_{n N_{2}}=\sum_{i=1}^{2 M+1} \pi_{0, i}$ $n=1,2,3 \ldots$

(3) Probability that the server is idle $=\pi_{0,1}$

(4) Probability that the server is busy $=1-\pi_{0,1}$

(5) The probability that there are zero customers in the virtual queue is $\pi_{0 N_{1}}=\sum_{i=0}^{\infty} \pi_{i, 1}$

(6) The probability that there are $\mathrm{n}$ customers in the virtual queue is

$\sum_{i=1}^{\infty}\left[\pi_{i, 2 n}+\pi_{i, 2 n+1}\right]$ $\pi_{n N_{1}}=\pi_{0, n+1}+$

(7) Length of invisible queue $=\sum_{i=0}^{\infty} i \pi_{i N_{2}}$

(8) Length of virtual queue $=\sum_{i=0}^{N} i \pi_{i N_{1}}$

(9) Blocking Probability [the probability that an entering customer is not allowed to get themselves registered $] \quad=\mathrm{P}\left(\mathrm{N}_{1}=\mathrm{M}\right)=\sum_{\mathrm{k}=1}^{\infty}\left[\pi_{\mathrm{k}, 2 \mathrm{M}}+\pi_{\mathrm{k}, 2 \mathrm{M}+1}\right]+$ $\pi_{0, \mathrm{M}+1}$

(10) Average number of customers in the system

$$
\begin{aligned}
& =E\left(N_{1}+N_{2}=n\right) \\
& =n\left\{\pi_{n, 1}+\sum_{k=1}^{n-1}\left[\pi_{n-k, 2 n-2}+\pi_{n-k, 2 n-1}\right]+\pi_{0, n+1}\right\}
\end{aligned}
$$




\section{NUMERICAL EXAMPLE \& RESULTS}

Consider an example to understand the performance measures of our model with $\lambda_{1}=2, \lambda_{2}=0.25, \mu=3$ and $\mathrm{M}=3$. The system is stable because $\frac{\lambda_{1}+\lambda_{2}}{\mu}=\frac{2.25}{3}=0.75<$ 1

Using the recursive principle of matrix geometric method, we get

$$
R^{(18)}=\left[\begin{array}{lllllll}
0.582 & 0.082 & 0.003 & 0.011 & 0.000 & 0.001 & 0.000 \\
0.047 & 0.581 & 0.011 & 0.050 & 0.002 & 0.006 & 0.000 \\
0.224 & 0.010 & 0.392 & 0.001 & 0.020 & 0.000 & 0.001 \\
0.254 & 0.055 & 0.101 & 0.390 & 0.015 & 0.019 & 0.001 \\
0.132 & 0.006 & 0.230 & 0.000 & 0.402 & 0.000 & 0.020 \\
0.000 & 0.000 & 0.000 & 0.000 & 0.000 & 0.559 & 0.000 \\
0.076 & 0.004 & 0.132 & 0.000 & 0.230 & 0.000 & 0.392
\end{array}\right]
$$

Solving for $\pi_{0}$ and $\pi_{1}$, and normalizing with $\alpha=1.3503$ we get

$\pi_{0}=\left[\begin{array}{llll}0.373 & 0.041 & 0.005 & 0.001\end{array}\right]$

$\pi_{1}=\left[\begin{array}{lllllll}0.239 & 0.034 & 0.021 & 0.005 & 0.01 & 0.001 & 0.012\end{array}\right]$

Calculating remaining $\pi_{i}$ using recursive formulae, we get

$\pi_{2}=\left[\begin{array}{lllllll}0.149 & 0.029 & 0.014 & 0.005 & 0.007 & 0.001 & 0.005\end{array}\right]$

$\pi_{3}=\left[\begin{array}{llllllll}0.095 & 0.022 & 0.009 & 0.004 & 0.004 & 0.001 & 0.002\end{array}\right]$

$\pi_{4}=\left[\begin{array}{llllllll}0.061 & 0.016 & 0.006 & 0.004 & 0.002 & 0.001 & 0.001\end{array}\right]$ :

\subsection{Performance Measures}

\begin{tabular}{|l|l|l|l|l|l|l|l|}
\hline $\begin{array}{l}\text { Stationary Probability for } \\
\text { number of customers }\end{array}$ & 0 & 1 & 2 & 3 & 4 & 5 & 6 \\
\hline Invisible & 0.42 & 0.322 & 0.21 & 0.137 & 0 & 0 & 0 \\
\hline Virtual & 0.856 & 0.17 & 0.04 & 0.023 & 0 & 0 & 0 \\
\hline Station & 0.373 & 0.28 & 0.209 & 0.154 & 0.056 & 0 & 0 \\
\hline
\end{tabular}

\begin{tabular}{|l|l|l|}
\hline & Length & $\begin{array}{l}\text { Blocking } \\
\text { Probability }\end{array}$ \\
\hline $\begin{array}{l}\text { Invisible } \\
\text { Queue }\end{array}$ & 1.153 & - \\
\hline $\begin{array}{l}\text { Visible } \\
\text { Queue }\end{array}$ & 0.319 & 0.023 \\
\hline System & 1.384 & - \\
\hline
\end{tabular}

\subsection{Choice of the truncation level $\mathrm{N}_{2}$}

$\mathrm{N}_{2}$ is the level of the QBD and its truncation can be considered as the number after which the probabilities are negligible and hence can be avoided. That is, we need $N_{2}$ such that $\sum_{n=N_{2}+1}^{\infty} \pi_{n} e<\varepsilon$. But this $N_{2}$ cannot be found as $\pi_{n}$ are unknown initially.

When the registered customers are not allowed, the model reduces to a regular $\mathrm{M} / \mathrm{M} / 1$ queue model whose arrival rate is $\lambda=\lambda_{1}+\lambda_{2}$. This system is stable because $\frac{\lambda}{\mu}<1$. Let $P_{n}$ be the probability of $\mathrm{n}$ customers in the system for $\mathrm{M} / \mathrm{M} / 1$ queue model.

$$
\text { Thus } P_{n}=\left(\frac{\lambda}{\mu}\right)^{n}\left(1-\frac{\lambda}{\mu}\right)
$$

Also as $\mathrm{M}$ increases we see from the numerical example that the tail probability of our model is lesser than the tail probability of M/M/1 queue model. That is, $\sum_{n=N_{2}+1}^{\infty} \pi_{n} e<$ $\sum_{n=N_{2}+1}^{\infty} P_{n}<\varepsilon$

This gives the choice of truncation of $N_{2}$.

In our numerical example it is observed that if we fix $\varepsilon=0.1$ then using M/M/1 model values we predict $N_{2}=3$ because $\sum_{n=4}^{\infty} P_{n}<\varepsilon$. One can observe that as predicted all remaining $\pi_{4}, \pi_{5} \ldots$ tend to zero in successive iterations.

\section{CONCLUSION}

We have suggested a new class of customers who register themselves to be called by the server. Using queueing theory concepts we have modeled the call centre and derived the stationary probabilities using matrix geometric method. This will no doubt increase the efficiency of service rendered to customers and results in customer satisfaction.

\section{REFERENCES}

1 Aguir M. S, Aksin .O. Z, Karaesmen. F and Dallery. Y, "On the interaction between retrials and sizing of call centres", European Journal of Operational Research 191, issue 2, 398-408, 2008.

2 Arul Freeda Vinodhini. G and Vidhya. V.,(2014), Transient solution of multi-server queue with catastrophes and Impatient customers when system is down, Applied Mathematical Sciences, 8:92, 4585- 4592.

3 Chakravarthy. S. R, Krishnamoorthy. A and Joshua V. C, "Analysis of a multiserver retrial queue with search of customers from the orbit, Performance Evaluation, 63, 776- 798, 2006

4 Choi B. D, Han D. H and Falin G. I, "On the virtual waiting time for an M/G/1 retrial queue with two types of calls", Journal of applied mathematics and Stochastic analysis, 6, 11-23, 1993

5 Gomez- Corral. A, "A matrix geometric approximation for tandem queues with blocking and repeated attempts", Operations research letters 30, 360-374, 2002.

6 Han D. H and Lee Y. W, "MMPP, M/G/1 retrial queue with two class of customers", Communication of the Korean Mathematical Society, 11, 481-493, 1996.

7 Lee Y. W, "The M/G/1 feedback retrial queue with two types of customers", Bulletin of Korean Mathematical society, 42, 875-887, 2005.

8 Neuts M.F, "Matrix Geometric solutions in Stochastic models: An algorithmic Approach", The Johns Hopkins University Press, Baltimore (1981)

9 Renganathan. N, Kalyanaraman. R and Srinivasan. B, "A finite capacity single server retrial queue with two types of calls", International Journal of Information and Mathematical Sciences, 13, No. 3, 47-56, 2002.

10 Vidhya.V, (2013), Disasters and Customer Impatience In Bulk Queueing Systems, Asia Pacific Journal of Research, Volume: I, Issue: VIII, 78-83. 